\title{
Rock Damage and Aquifer Property Estimation from Water Level Fluctuations in Wells Induced by Seismic Waves: A Case Study in X10 Well, Xinjiang, China
}

\author{
Di Zhao $\mathbb{B D}^{1,2,3}$ Yifan Zeng, ${ }^{1,3}$ Xiaolong Sun, ${ }^{4}$ and Aoshuang Mei ${ }^{1,2,3}$ \\ ${ }^{1}$ China University of Mining and Technology, Beijing 100083, China \\ ${ }^{2}$ State Key Laboratory of Biogeology and Environmental Geology, China University of Geosciences, Beijing 100083, China \\ ${ }^{3}$ National Engineering Research Center of Coal Mine Water Hazard Controlling, Beijing 100083, China \\ ${ }^{4}$ National Institute of Natural Hazards, Ministry of Emergency Management of China, Beijing 100085, China
}

Correspondence should be addressed to Di Zhao; 527753078@qq.com

Received 5 October 2021; Accepted 13 November 2021; Published 2 December 2021

Academic Editor: Fan Deyuan

Copyright ( 92021 Di Zhao et al. This is an open access article distributed under the Creative Commons Attribution License, which permits unrestricted use, distribution, and reproduction in any medium, provided the original work is properly cited.

\begin{abstract}
There is a coupling relationship between surrounding rock stress, deformation, and fracture evolution, especially in the microdynamics of the crust caused by mining activities and earthquakes. Previous research has investigated many cases regarding the coseismal water level responses and proposed a method to calculate the aquifer parameters by tidal analysis. However, to date, measurement of the degree of rock damage in the field has not been reported. Quantifying the fracture characteristics is essential for accurate evaluation of rock stability. This study has analyzed the relationship between the seismograms and hydroseismograms in response to the $M w 7.8$ Solomon Islands earthquake and the $M w 7.8$ Kaikoura earthquake, both events occurring in 2016. The calculated and measured changes in water level in the X10 well were fitted in order to study the relationships among the volumetric strain, the deviatoric strain, and the oscillations in the pore pressure. Then, we further estimate the degree of rock damage and the hydraulic characteristics of the aquifer. The results showed that the values for the rock damage parameter, $0.662<\alpha_{\mathrm{D}}<0.754$, and the Skempton coefficient, $-0.100<A<0.026$, estimated for the Solomon Islands earthquake signified higher damage and dilatancy in the X10 well. Also, the respective values for the parameters, $0.293<\alpha_{\mathrm{D}}<0.363$ and $0.226<A<0.251$, calculated for the Kaikoura earthquake signified a lower degree of rock damage. It is concluded that the changes in the pore pressure were influenced by both the volumetric strain and the deviatoric strain. The degree of rock damage and the hydraulic properties of the aquifer estimated from the water level fluctuations in the wells which were induced by the seismic waves represent the actual aquifer characteristics.
\end{abstract}

\section{Introduction}

Understanding the mechanical and hydrological properties of rock is important for engineering, including mining, sources evaluation, landslide stability assessment, infrastructure stability, reservoir geomechanics, and so on [1-4]. Fractures significantly control the properties of rock, and thus quantifying fracture characteristics is essential for accurate evaluation of rock stability. Usually, an empirical damage-mechanism model is introduced and is widely used in geophysics and engineering for irreversible deformation [5]. The rock damage is described by $0<\alpha_{\mathrm{D}}<1$ which is influenced by aquifer properties and stress state [6]. High damage value $\left(\alpha_{\mathrm{D}}>0.8\right)$ means that the rock is highly fractured and its mechanical properties are low while its permeability is high. Low damage value $\left(\alpha_{\mathrm{D}}<0.2\right)$ means stiff and strong rock with low permeability. However, measuring the rock damage in the field is still impossible.

Water level influenced by barometric pressure, Earth tides, and earthquakes indicates that aquifer is sensitive to the crustal strain which provides a way to probe the material characteristics [7-12]. It is generally recognized that the water level in aquifers responds to volumetric strain $[13,14]$. Moreover, the water level responses in aquifer system caused by earthquakes may be explained by the dynamic interactions between the seismic waves and the aquifers $[15,16]$. 
The Rayleigh and $P$ waves are the seismic waves that have been documented most widely as being responsible for the expansion and contraction of aquifers [17-19]. However, recent studies have shown that the water level of well also responds to $S$ and Love waves because of the anisotropic poroelastic characteristics $[15,20]$. Laboratory studies on the effect of shear stress on pore pressure demonstrated that the deviatoric strain significantly influenced the pore pressure, and there was a strong correlation as the degree of rock damage increased [21, 22]. Recently, Shalev et al. [23] showed that the water level in wells responding to deviatoric strain depended on the rock damage, and the deviatoric strain of the damaged rock led to high amplitudes in the water level. For rocks without damage, there was no response from the water level in the well [23]. Therefore, it should, in principle, be possible to determine quantitatively the degree of damage to the rock from the responses of the water level to the deviatoric strain. In this study, the relationship between the seismograms and the hydroseismograms in response to the $M w 7.8$ Solomon Islands earthquake (2016-12-9) and the Mw 7.8 Kaikoura earthquake (2016-11-13) will be investigated. The X10 well in Xinjiang is selected for study because it is located at the intersection of two faults with extensive rock fractures. The rock damage and the hydraulic properties of the aquifer in the well will be estimated from the fluctuations in the water level induced by the seismic waves.

\section{Observational Background and Hydrogeological Setting}

The groundwater well (X10) was established to the south of Urumqi City in Xinjiang of China by the China Earthquake Administration in September 1980 to monitor the possible reservoir-induced seismicity. It is located at the intersection of Liushugou-Hongyanchi fault $\left(43.70^{\circ} \mathrm{N}, 87.62^{\circ} \mathrm{E}\right.$; with the elevation of $1056 \mathrm{~m}$ above sea level) (Figure 1). The depth of the open well is $28 \mathrm{~m}$. The aquifer is located in the depth between 16 and $24 \mathrm{~m}$, consisting of sandstone and muddy siltstone of Permian Hongyanchi group. It has a static water of approximately $1 \mathrm{~m}$ below ground surface. Extensive fractures could be found, and a screen section was installed.

\section{Seismic Data}

The water level in the well was recorded using an SWY-II type digital meter from October 2016 to the present with an accuracy of $\pm 0.05 \%$ F.S. and a precision of $\pm 0.02 \%$ F.S. in the range of $0-50 \mathrm{~m}$ [16]. The sampling interval was $1 \mathrm{~s}$, and the information recording capability was a significant improvement. Furthermore, an SLJ-100 type three-component seismograph was used for collecting the radial, transverse, and vertical components of the seismograms following the earthquakes. The hydroseismograms and the seismograms following the Mw 7.8 Solomon Islands earthquake and the Mw 7.8 Kaikōura earthquake are shown in Figure 2. The epicenter of the Solomon Islands earthquake was $9,543 \mathrm{~km}$ southeast of the X10 well while the epicenter of the Kaikoura earthquake was $12,798 \mathrm{~km}$ southeast of the well.
The X10 well is connected indirectly to the aquifer through the fault. The water flow in the pores of the aquifer may flow indirectly to the well bore along the fracture channel of the fracture zone. Taking into account the arrival time of the P, S, Love, and Rayleigh waves, the data of 1 : 50-3:05 (GMT +8:00) (Solomon Islands earthquake) and 18:43-19:58 (GMT +8:00) (Kaikōura earthquake) were selected for this study. Spline interpolation was used to fill in the missing data, and the data were smoothed by a high-pass Butterworth filter with a bottom corner frequency of $0.01 \mathrm{~Hz}$ to remove the long period trends. The times before the arrival of the seismic waves-1:50:00-2:00:42 on 2016-12-9 (Solomon Islands earthquake) and $18: 43: 00-19: 16: 28$ on 2016-11-13 (Kaikōura earthquake) - were regarded as the noninterference states. The periods of $2: 00: 43-2: 10: 42$ or $2: 10: 43-2: 20: 00$ for the Solomon Islands earthquake corresponding to $\mathrm{P}, \mathrm{S}$, Love, and Rayleigh waves, respectively, were regarded as the interference state, and the same was considered for the periods $19: 16: 29-19: 27: 37$ or 19 : $27: 38-19: 38: 04$ in the case of the Kaikōura earthquake. When the long period waves of Love and Rayleigh arrived, the largest amplitudes for the level of the well water occurred [24]. Compared with the P and S period, the water level response was more distinct during the Love wave period and Rayleigh wave period. The vertical and radial seismograms were larger than those in the transverse direction. The shapes of radial, transverse, and vertical seismograms were similar to those of water level changes. Because of the imbalance of the total water head between the wellbore and the aquifer caused by air pressure, the response of the water level lagged behind that of the seismic waves. This phenomenon was attributed to the wellbore storage effect, which could cause attenuation of the water level, the water pressure, the phase shift, and the aquifer permeability $[7,8,13,17,18,25,26]$. Due to wellbore storage effect, the measured changes in the water level are not accurate. In order to maintain equilibration in the aquifer-borehole, when estimating the rock failure by changes in the water level, it is necessary to correct for the wellbore storage effect. Such a correction method has been reported by Liu et al. [18]. Also, the attenuation factor of an open well can be calculated from equations $[4,17,18]$. The attenuation factor depends mainly on the transmissivity, the storage coefficient, the aquifer thickness, and the effective height of the water column in the well-aquifer system. In this study, the attenuation factor of the X10 well was 2.049 as calculated for the Solomon Islands earthquake while the value was 8.967 for the Kaikōura earthquake.

\section{Methods}

4.1. Estimation of the Degree of Rock Damage by Seismic Analysis. The amplitudes of the oscillations in the water level and the changes in the pore pressure are affected by the wavelength and frequency of the specific seismic waves. Poroelastic theory has been used extensively to study the oscillations in the water level in response to earthquakes, barometric pressure, and Earth tides, especially with respect to the seismicity in oil-rich areas [27-29]. Several studies have successfully applied poroelastic theory in explaining the 

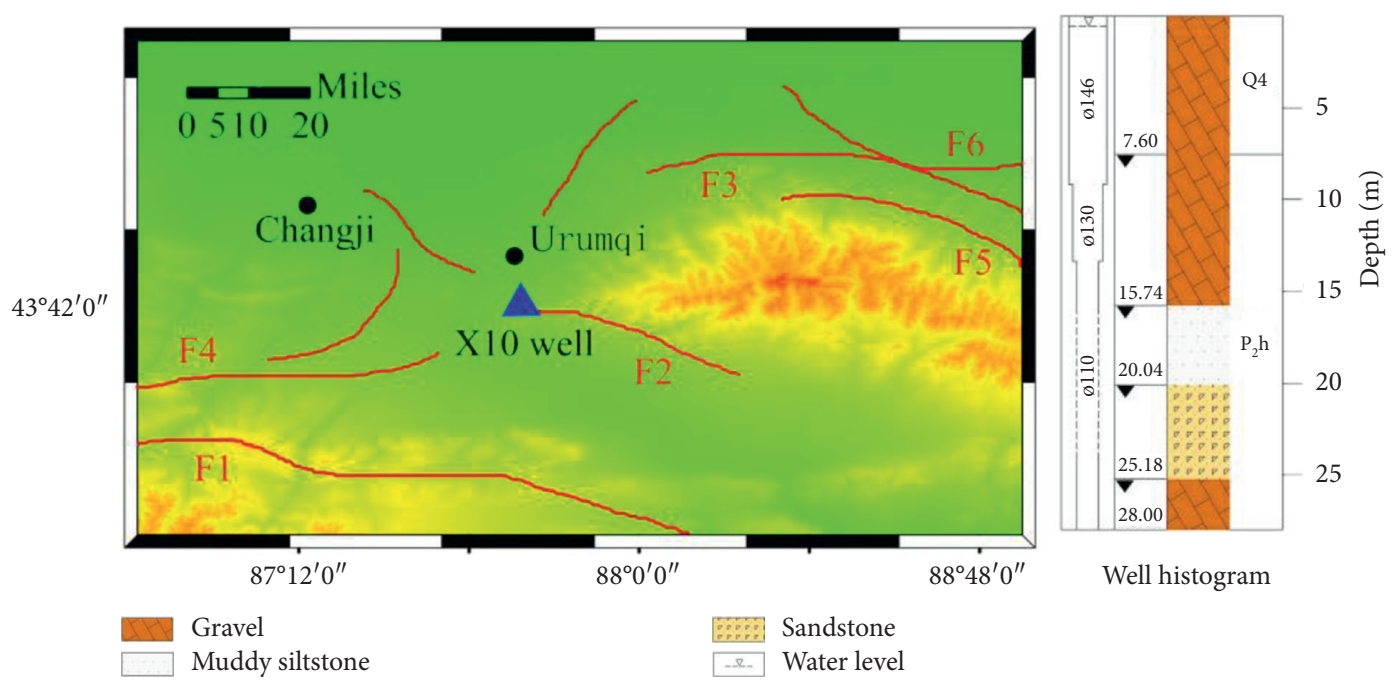

FIgURE 1: Location of the X10 well and stratigraphy of the borehole [16]. F1 : southern margin fault of Chaiwopu Basin; F2: Hongyanchi Fault; F3: Yama-Kerrey Fault; F4: Xishan Fault; F5: Erdaogou Fault; F6: Fukang Fault.
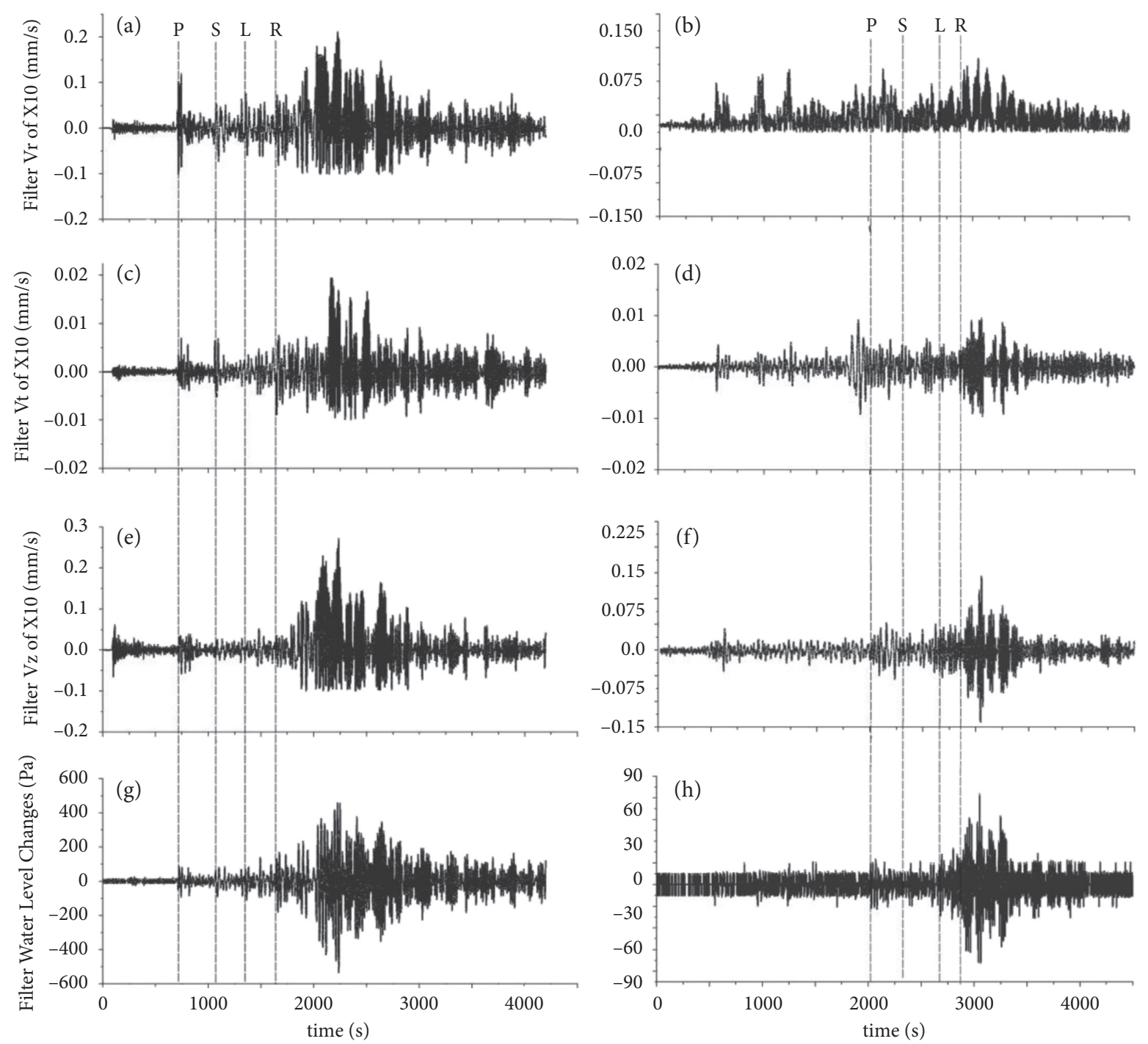

FIGURE 2: Comparison of hydroseismograms and three-component seismograms from 1:50 to 3:05 (GMT +8:00) on 9 December 2016 of the Mw7.8 Solomon Islands earthquake and from 18:43 to 19:58 (GMT +8:00) on 13 November 2016 of the (M)w7.8 Kaikoura earthquake. (a, b) Radial seismograms. (c, d) Transversal seismograms. (e, f) Vertical seismograms. (g, h) Water level changes. Annotation marks the arrivals of $\mathrm{P}, \mathrm{S}, \mathrm{L}$, and $\mathrm{R}$ waves. 
relationship between the well-aquifer system and seismic waves $[16,19,23]$. Poroelastic theory attested to the fact that the strain was affected by the both pore pressure and stress in an isotropic porous elastic medium [30]. According to Henkel and Wade [31], Holtz et al. [32], and Skempton [33], changes in pore pressure were also affected by the deviatoric strain [31-33]. It was reported that based on Biot's theory, changes in the pore pressure could not be caused by deviatoric stress [34]. However, Skempton [33] allowed for this possibility under undrained conditions. A two-dimensional stress state was employed to describe the relationship between the deviatoric strain, the volumetric strain, and the pore pressure [23]:

$$
\Delta P f=B K_{u} \varepsilon_{\mathrm{v}}+N \varepsilon_{\mathrm{d}},
$$

where $\triangle P_{\mathrm{f}}$ represents the change in pore pressure, $K_{\mathrm{u}}$ is the undrained bulk modulus [35], $\varepsilon_{\mathrm{v}}$ is the volumetric strain, $N$ is the coupling coefficient of the shear strain, $N=-4 \mu B(A-1 / 3)$, $\mu$ is the shear modulus, and $\varepsilon_{\mathrm{d}}$ is the deviatoric strain where $\varepsilon_{\mathrm{d}}=\left(\varepsilon_{1}-\varepsilon_{3}\right) / 2$. $A$ and $B$ are referred to as Skempton coefficients which relate to rock compressibility. $B$ depends on the rock lithology in the range from 0 to 1 . A may be expressed as follows:

$$
A=\frac{1}{3}-\frac{4 K_{u} \gamma}{4(2 \mu+\gamma) \gamma-3(6 \mu+\gamma)\left(K_{u}+4 / 3 \gamma\right)} .
$$

$A$ is related to $\mu$ and an additional modulus $\gamma$ which couples the shear and volumetric stress of the nonlinear materials. $\mu=\mu_{0}+\alpha_{\mathrm{D}} \zeta_{0} \gamma$, and $\gamma=\alpha_{\mathrm{D}} \gamma_{\Gamma}$, where $\mu_{0}, \gamma_{\Gamma}$, and $\zeta_{0}$ are the elastic moduli. $\zeta_{0}$ is a critical value of the strain invariant ratio at the onset of damage accumulation and is connected to the internal friction angle of the friction law for rocks. $\zeta_{0}=-1$, which is based on the typical value for sandstones [8]. The damage parameter $\alpha_{D}$ represents the elastic characteristics of a damaged solid.

Substituting $N=-4 \mu B(A-1 / 3)$ into (2) gives the value for $N$ :

$$
N=\frac{8 B K_{u} \gamma(2 \mu+\gamma)}{4(2 \mu+\gamma) \gamma-3(6 \mu+\gamma)\left(K_{u}+4 / 3 \gamma\right)},
$$

where the tidal coefficient $B K_{\mathrm{u}}$ is a constant. It is characterized by rock elasticity. The $\alpha_{\mathrm{D}}$ value is equal to 1 for a material completely damaged and equal to 0 for a damagefree material.

The relationship between the volumetric strain, the deviatoric strain, and the pore pressure can be established by both tidal analysis and seismic analysis. Then, the rock damage may be calculated.

4.2. Estimation of Parameters by Tidal Analysis. In a previous study by Doan and Cornet [36], the relationship between the phase shift and the poroelastic properties was used to determine $B$ and $K_{\mathrm{u}}$. In addition, the permeability and transmissivity may be estimated by tidal analysis $[7,37]$.
In a well, when the poroelastic medium of the rock changes due to external force, the water level will fluctuate. The relationship between volumetric strain and pore pressure may be expressed as follows:

$$
p=B K_{u} \varepsilon
$$

where $P$ is the pore pressure, $\varepsilon$ is the strain, $B K_{\mathrm{u}}$ is characterized by the rock elasticity, and $B$ depends on the rock lithology (the value is in the range of 0 to 1 ).

$$
p=\rho g h \text {. }
$$
follows:

Substituting (5) into (4), BKu may be expressed as

$$
B K_{u}=\rho g \frac{h}{\varepsilon}
$$

where $h / \varepsilon$ is the tidal factor, which represents the fluctuations in the amplitude ratio of the water level to volumetric strain, $\rho$ is the water density, $\rho=1 \mathrm{~g} / \mathrm{cm}^{3}, g$ is the acceleration due to gravity, $h$ is the water level, and $\varepsilon$ is the volumetric strain.

In an ideal, confined, and isotropic well-aquifer system, the parameters can also be estimated by tidal analysis $[7,38]$ :

$$
\frac{\Delta h}{\Delta \varepsilon}=-\frac{1}{S_{s}},
$$

where $\Delta h / \Delta \varepsilon$ is the tidal factor, $\Delta h$ is the change in water level, $\Delta \varepsilon$ is the change in the volumetric strain, and $S_{s}$ is the specific storage. $\Delta h / \Delta \varepsilon$ may be determined from tidal analysis. According to (7), the tidal factor and the specific storage obey a reciprocal relationship. Moreover, the relationship between $S_{\mathrm{s}}$ and $n$ is as follows:

$$
n=\frac{B_{e} S_{s}}{\beta \rho}
$$

where $n$ is the porosity, $B_{\mathrm{e}}$ is the barometric efficiency calculated by a regression deconvolution method or Clark's method $[16,25]$, and $\beta$ is the water compressibility, $\beta=4.6 \times 10^{-10} \mathrm{~m}^{2} / \mathrm{N}$. Lithology dilatancy is an important characteristic for granules especially for dense sand. An increase in $n$ represents an increase in the expansion of the lithology [39].

\subsection{Estimation of Parameters by Seismic Analysis}

4.3.1. Strain in Surface Waves. The actual state of rock failure during the deformation process is difficult to estimate. Hence, this research is meaningful for studying the formation of old ruptures and the generation of new ruptures. Love and Rayleigh waves propagate along the surface of the Earth, and they can change in their propagation direction and attenuate with depth. Also, stress can be estimated from the seismic wave velocities: 


$$
\begin{gathered}
\varepsilon_{v}=\frac{1-2 v_{u}}{1-v_{u}} \frac{\partial_{U R}}{\partial_{R}} \\
=\frac{1-2 v_{u}}{1-v_{u}} \frac{v_{R}}{V_{R, L}}, \\
\varepsilon_{d}=\frac{1}{2} \frac{\partial_{U T}}{\partial_{R}} \\
=\frac{1}{2} \frac{v_{T}}{V_{R, L}},
\end{gathered}
$$

where $u_{\mathrm{R}}$ is the radial displacement, $V_{R}$ is the radial seismic wave velocity, $u_{\mathrm{T}}$ is the transverse displacement, and $V_{T}$ is the transverse seismic wave velocity. $V_{\mathrm{R}}(3.5 \mathrm{~km} / \mathrm{s})$ is the velocity of the Rayleigh wave, and $V_{\mathrm{L}}(3 \mathrm{~km} / \mathrm{s})$ is the velocity of the Love wave, which depends on the particular phase $[23,40]$.

Combining (9), (10), and (1), the following formula is obtained:

$$
\Delta P_{f}=B K_{u} \frac{1-2 v_{u}}{1-v_{u}} \frac{v_{R}}{V_{R, L}}+N \frac{1}{2} \frac{v_{T}}{V_{R, L}} .
$$

When only the effect of the volumetric strain on the pore pressure is considered [23]:

$$
\Delta P_{f}=B K_{u} \frac{1-2 v_{u}}{1-v_{u}} \frac{v_{R}}{V_{R, L}} .
$$

When only the effect of the deviatoric strain on the pore pressure is considered:

$$
\Delta P_{f}=N \frac{1}{2} \frac{v_{T}}{V_{R, L}} .
$$

4.3.2. Strain in Body Waves. The volumetric strain and the deviatoric strain calculated for body waves are as follows:

$$
\begin{aligned}
\varepsilon_{v} & =\frac{\partial u_{z}}{\partial Z} \\
& =\frac{v_{z}}{V_{P, S}}, \\
\varepsilon_{d} & =\frac{1}{2} \frac{\partial u_{T}}{\partial Z} \\
& =\frac{1}{2} \frac{v_{T}}{V_{P, S}},
\end{aligned}
$$

where $u_{\mathrm{z}}$ is the vertical displacement and $v z$ is the vertical velocity $^{23} . V_{\mathrm{p}}(2.5 \mathrm{~km} / \mathrm{s})$ is the velocity of the $P$ wave and $V_{\mathrm{s}}$ $(1.5 \mathrm{~km} / \mathrm{s})$ is the velocity of the $S$ wave, which are dependent on the particular phase $[23,40]$. Both phases are the acknowledged seismic waves which can lead to aquifer expansion and contraction [41].

Substituting (14) and (15) into (1) results in the following equation:

$$
\Delta P_{f}=B K_{u} \frac{v_{z}}{V_{P, S}}+N \frac{1}{2} \frac{v_{T}}{V_{P, S}} .
$$

\section{Results}

5.1. Tidal Analysis. In this study, the Solomon Islands earthquake and the Kaikoura earthquake were selected. The time series selected was divided into 10-day interval and a 5day sliding window. The tidal factors obtained by BAYTAP$G$ in each interval did not change with time, which indicated that the aquifer characteristics were stable. The tidal factor estimated for the Solomon Islands earthquake was $4.167 \times 10^{5} \mathrm{~m}$, and that for the Kaikōura earthquake was $4.831 \times 10^{5} \mathrm{~m}$.

In the Solomon Islands earthquake, $B K_{\mathrm{u}}$ was $4.167 \mathrm{GPa}$ as calculated by (6). Considering the negative reciprocal relationship of the specific storage and the tidal factor, a value of $S_{\mathrm{s}}=2.40 \times 10^{-6} \mathrm{~m}^{-1}$ was obtained. Meanwhile, $n=0.27$ was estimated. For the Kaikōura earthquake, $B K_{\mathrm{u}}=4.831 \mathrm{GPa}, \quad S_{\mathrm{s}}=2.07 \times 10^{-6} \mathrm{~m}^{-1}, \quad$ and $n=0.22$, as specified in Table 1.

\subsection{Seismic Analysis}

5.2.1. The Love and Rayleigh Phases. In this study, the time series of the Love and Rayleigh phases during the Solomon Islands earthquake $(2: 10: 43-2: 20: 00)$ and the Kaikōura earthquake $(19: 27: 38-19: 38: 04)$ were selected. Using the seismic velocities, the measured water level changes and the calculated water level changes were fitted. The results are listed in Table 2 . When assuming only volumetric strain, $B K_{u}\left(1-2 v_{u}\right)\left(1-v_{u}\right)=1.304 \mathrm{GPa}, B K_{\mathrm{u}}=3.616 \mathrm{GPa}$ with a correlation coefficient of $R^{2}=0.675$ for the Solomon Islands earthquake (Figure $3(\mathrm{a})$ ) and $B K_{\mathrm{u}}=12.364 \mathrm{GPa}$ with a correlation coefficient of $R^{2}=0.001$ for the Kaikoura earthquake (Figure 3(b)). When assuming only deviatoric strain, $N=17.363 \mathrm{GPa}$ with a low correlation coefficient value of $R^{2}=0.258$ for the Solomon Islands earthquake (Figure 3(c)) and $N=2.529 \mathrm{GPa}$ with $R^{2}=0.557$ for the Kaikōura earthquake (Figure $3(\mathrm{~d})$ ). The relatively smaller $R^{2}$ value for the former indicated that only the volumetric or the deviatoric strain in the X10 well had small effects on the changes in the pore pressure. When considering both the volumetric strain and the deviatoric strain, $B K_{u}\left(1-2 v_{u}\right)\left(1-v_{u}\right)=1.165 \mathrm{GPa}, B K_{\mathrm{u}}=3.229 \mathrm{GPa}$, and $N=8.437 \mathrm{GPa}$ with $R^{2}=0.728$ (Solomon Islands earthquake) (Figure 3(e)) and $B K_{u}\left(1-2 v_{u}\right)\left(1-v_{u}\right)=1.439 \mathrm{GPa}$, $B K_{\mathrm{u}}=3.717 \mathrm{GPa}$, and $N=2.529 \mathrm{GPa}$ with $R^{2}=0.560$ (Kaikōura earthquake) (Figure 3(f)). The best correlation coefficient between the calculated and measured water pressure showed that the changes in the pore pressure were influenced by both the volumetric strain and the deviatoric strain.

5.2.2. The $P$ and $S$ Phases. When considering both the volumetric strain and the deviatoric strain of the $P$ and $S$ phases, the results were $B K_{\mathrm{u}}=0.397 \mathrm{GPa}$ and $N=7.384 \mathrm{GPa}$ with $R^{2}=0.554$ for the Solomon Islands earthquake (Figure $3(\mathrm{~g})$ ) and $B K_{\mathrm{u}}=3.197 \mathrm{GPa}$ and $N=2.238 \mathrm{GPa}$ with $R^{2}=0.201$ for the Kaikōura earthquake (Figure $3(\mathrm{~h})$ ). The amplitude during the $P$ and $S$ periods was small, and many 
TABLE 1: Well-aquifer system parameters by $M_{2}$ wave tidal.

\begin{tabular}{lcccc}
\hline Earthquake & $B K_{\mathrm{u}}(\mathrm{GPa})$ & $S_{\mathrm{s}}\left(\mathrm{m}^{-1}\right)$ & $B_{\mathrm{e}}$ & $\beta\left(\mathrm{m}^{2} / \mathrm{N}\right)$ \\
\hline Kaikoura earthquake & 4.831 & $2.07 \times 10^{-6}$ & 0.4889 & $4.6 \times 10^{-10}$ \\
Solomon Islands earthquake & 4.167 & $2.40 \times 10^{-6}$ & 0.5036 & $4.6 \times 10^{-10}$ \\
\hline
\end{tabular}

TABle 2: Well-aquifer system parameters by seismic analysis.

\begin{tabular}{lcccccc}
\hline \multirow{2}{*}{ Earthquake } & \multicolumn{2}{c}{ Volumetric strain } & \multicolumn{2}{c}{ Deviatoric strain } & \multicolumn{3}{c}{ Volumetric strain and deviatoric strain } \\
& $B K_{\mathrm{u}}(\mathrm{GPa})$ & $R^{2}$ & $N(\mathrm{GPa})$ & $R^{2}$ & $B K_{\mathrm{u}}(\mathrm{GPa})$ & $N(\mathrm{GPa})$ \\
\hline Kaikoura earthquake & 12.364 & 0.001 & 2.529 & 0.557 & 3.717 & 2.529 \\
Solomon Islands earthquake & 3.616 & 0.675 & 17.363 & 0.258 & 3.229 & 0.560 \\
\hline
\end{tabular}

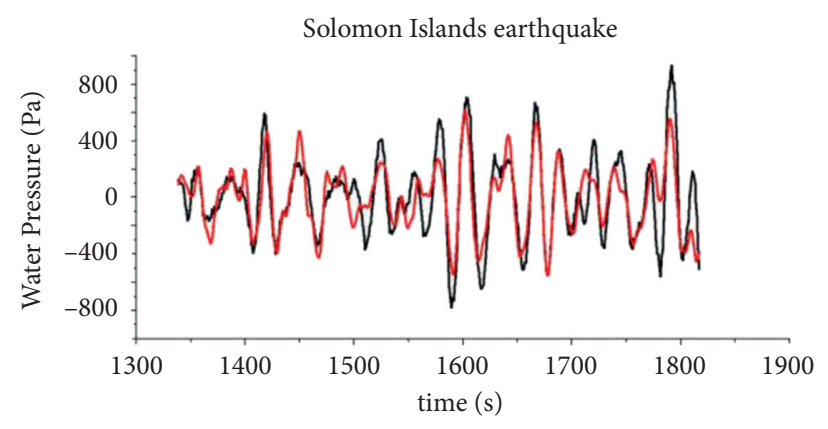

- Observed Water Pressure

_ Calculated Water Pressure

(a)

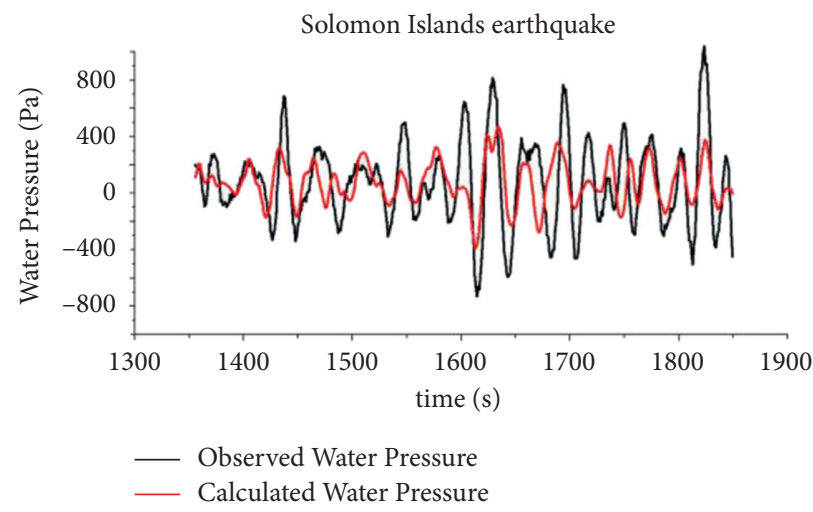

(c)

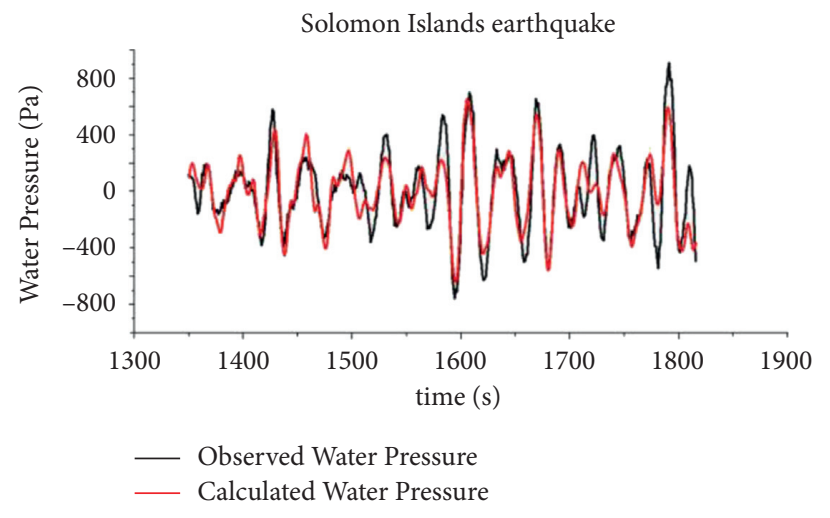

(e)

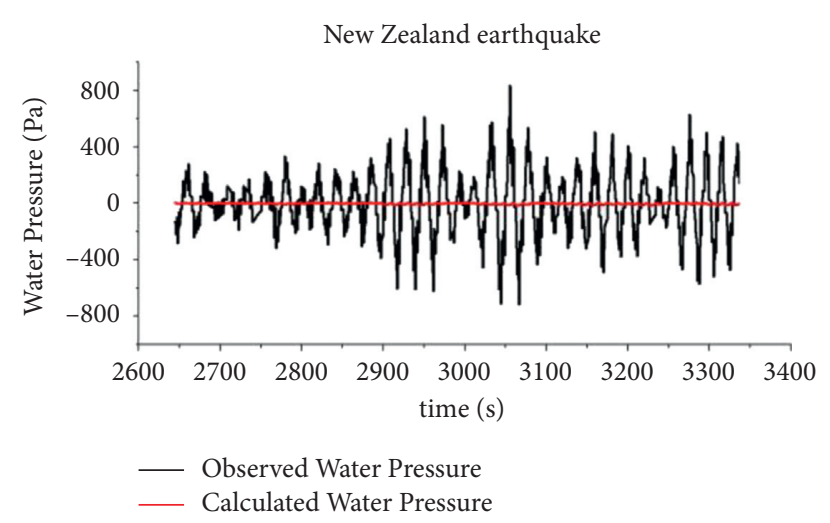

(b)

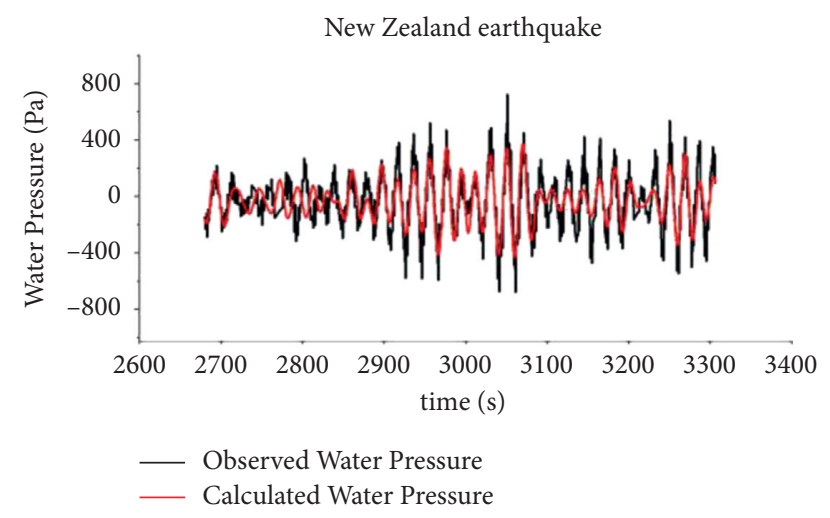

(d)

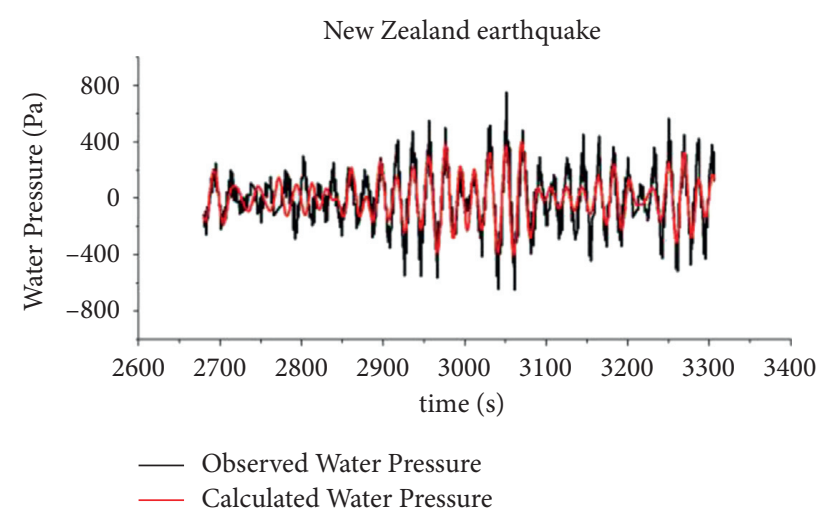

(f)

Figure 3: Continued. 


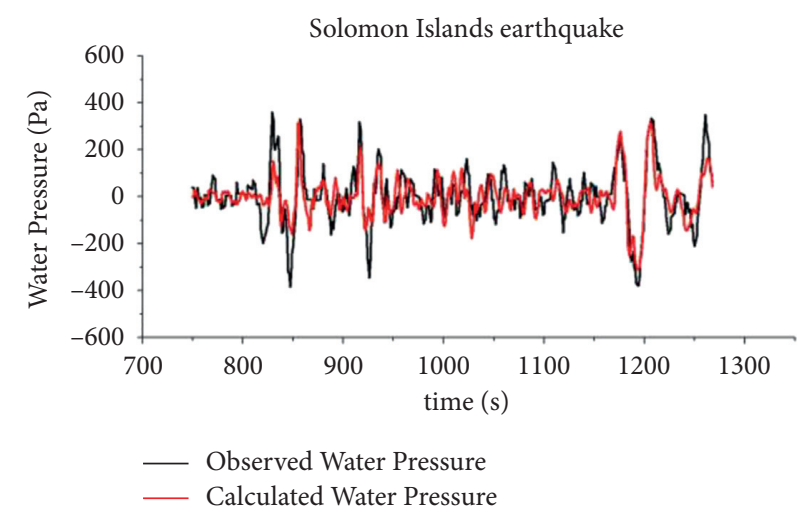

(g)

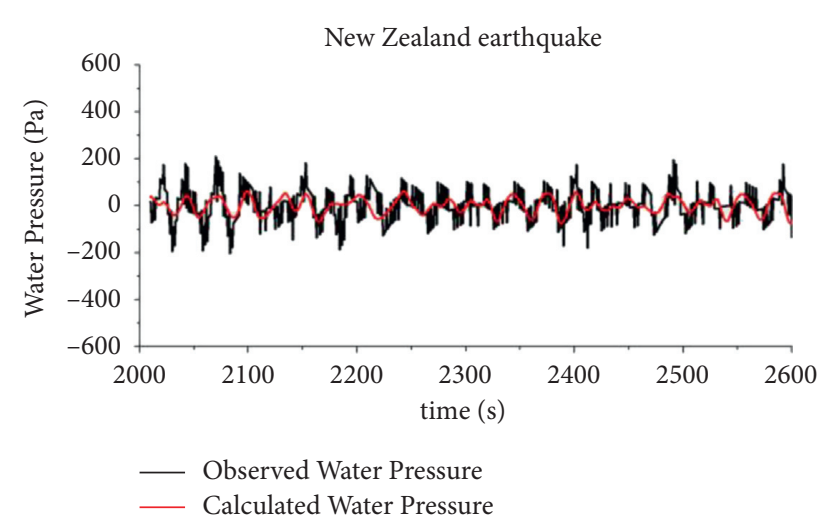

(h)

FIGURE 3: Calculated and observed water pressure in X10 in response to Solomon Islands earthquake and Kaikōura earthquake: (a, b) assuming only volumetric strain; (c, d) assuming only deviatoric strain; (e, f) assuming both volumetric strain and deviatoric strain for surface waves; $(\mathrm{g}, \mathrm{h})$ assuming both volumetric strain and deviatoric strain for body waves.

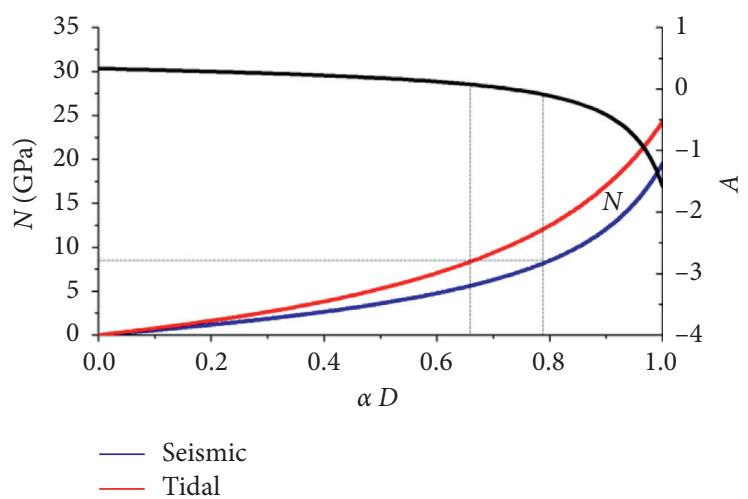

(a)

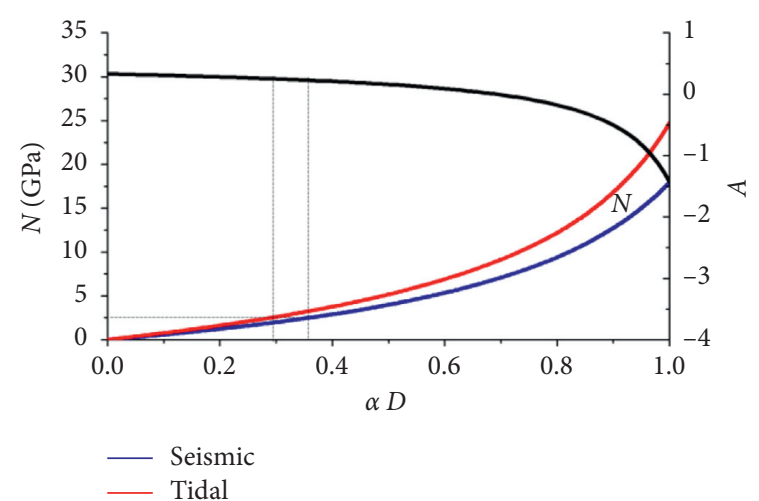

(b)

FIgURE 4: The deviatoric strain coupling: $A$ and $N$, as a function of rock damage $\left(\alpha_{\mathrm{D}}\right)$ for the seismic and tidal analysis. (a) Solomon Islands earthquake. (b) Kaikōura earthquake.

phases were contained in the same packet, so the estimated strain by the $P$ and $S$ waveforms was not well resolved. Surface waves can cause significant expansion of the aquifer compared to that of the body waves. Therefore, the pressure fluctuations were caused mainly by surface waves, and the parameters should be calculated with reference to the Love and Rayleigh phases.

\section{Discussion}

6.1. Estimation of Parameters. The obtained $B K_{\mathrm{u}}$ values for the two earthquakes by tidal analysis were similar $(4.167 \mathrm{GPa}$ for the Solomon Islands earthquake and $4.831 \mathrm{GPa}$ for the Kaikōura earthquake) while the respective values were 3.229 GPa (Solomon Islands earthquake) and $3.717 \mathrm{GPa}$ (Kaikōura earthquake) by seismic analysis. Zhang and Huang [42] showed that the $B K_{\mathrm{u}}$ values for sandstone measured in the laboratory ranged from 0.039 to $26.40 \mathrm{GPa}$. Also, Liao et al. [43] reported the measurement of $B K_{\mathrm{u}}$ values at different effective pressures in overseas trials. These independent studies demonstrated that the results of this study were consistent with real-world data. In addition, according to the experimental data for Berea Sandstone under a mean effective stress of $10 \mathrm{MPa}, B K_{\mathrm{u}}$ varied from 8.5 to $15.5 \mathrm{GPa}$ [30]. $B K_{\mathrm{u}}$ for the $\mathrm{X} 10$ well obtained in this study was very close to the range for sandstone in the experimental examples. Therefore, the rock damage and the aquifer properties estimated from the water level fluctuations in wells induced by seismic waves represent the actual aquifer characteristics. Considering the parameter inversion results and the different $B K_{\mathrm{u}}$ values from previous studies, it is concluded that there is good agreement between the seismic analysis and the tidal analysis, and this is in accordance with the experimental results for sandstone. The best correlation coefficient between the calculated and measured water pressure and the water level fluctuations indicated that the pore pressure was influenced by both the volumetric strain and the deviatoric strain. 
6.2. Estimation of Rock Damage. A linear poroelastic material without dilatancy has $A=1 / 3$ and $N=0$ and an undrained Poisson's ratio $V_{\mathrm{u}}=0.25$. It is known that stress expansions exist in fractured rock [39]. For porous sandstone without cohesion under undrained conditions, with stress increasing, the sensitivity of pore pressure to deviatoric strain increases [21]. With accumulation of the rock damage, the coupling between the pore pressure and strain becomes increasingly apparent, and the shear stress coupling coefficient increases. This situation tends to appear in active tectonic areas which have high levels of stress. Thus, in combining the tectonic and geological conditions, the high stress coefficient values mean that the rocks around the well are close to damage [23]. In this study, $N$ was calculated by (3). Based on the response of the pore pressure to stress, the damage parameter $\alpha_{\mathrm{D}}$ and the Skempton coefficient $A$ can be estimated by (2) (Figure 4).

From the results of seismic analysis, the shear stress coupling coefficient $N=8.437 \mathrm{GPa}$ corresponded to $\alpha_{\mathrm{D}}$ values of 0.662 to 0.754 , and $A$ values for this damage state were between -0.100 and 0.026 in the X10 well as estimated for the Solomon Islands earthquake (Figure 4(a)); also, $N=2.529 \mathrm{GPa}$ which corresponded to respective values of $0.293<\alpha_{\mathrm{D}}<0.363$ and $0.226<A<0.251$ as estimated for the Kaikoura earthquake (Figure 4(b)). The high shear stress on the faults signified that the strain was large enough to lead to remarkable water level oscillations.

6.3. The Degree of Rock Damage. Shalev et al. [23] showed that the response of the water level to deviatoric strain depended on the rock damage and that the deviatoric strain of the damaged rock led to high amplitudes in the water level. In the undamaged state, there is no response [23]. In analytical studies, a coupling of $A=0.2$ related to $\alpha_{\mathrm{D}}=0.45$ near Parkfield was considered to be slightly below $A=1 / 3$ which indicated that there was no obvious dilatancy in the linear poroelastic medium [44]. In contrast, in the Gome 1 well established on the Dead Sea Transform, $B K_{\mathrm{u}}$ values of $14 \mathrm{GPa}$ and $11 \mathrm{GPa}$ were calculated by tidal analysis and seismic analysis, respectively, for the condition $V_{\mathrm{u}}=0.38-0.4$. The final result was $\alpha_{\mathrm{D}}=0.84-0.9$ [23]. According to an experiment whereby a deviatoric stress of $65 \mathrm{MPa}$ with a confining pressure of $17 \mathrm{MPa}$ was imposed to sandstones with $n=21 \%$ in Berea and a deviatoric stress of $68 \mathrm{MPa}$ with a confining pressure of $17 \mathrm{MPa}$ was imposed to sandstones with $n=22 \%$ in Navajo, the conclusion was that an increasing $A$ meant an increase in the original rupture and the production of a new rupture[21].

In this research, it was found that stress would cause the porous elastic media parameters to change which signified that the rock was damaged. The results for $\alpha_{\mathrm{D}}$ and $A$ suggested different degrees of rock damage in the X10 well during the two earthquake periods: $0.662<\alpha_{\mathrm{D}}<0.754$ and $-0.100<A<0.026$ values estimated for the Solomon Islands earthquake (2016-12-9) were indicative of higher damage and dilatancy in the linear poroelastic medium of the X10 well.
Sun et al. [16] proposed a methodology that exploited spectral analysis based on the response of the water level fluctuations to Rayleigh waves to calculate the hydraulic parameters. Using this method, the optimal hydraulic conductivity $K$ was $324 \mathrm{~m} / \mathrm{d}$ for the Solomon Islands earthquake and $69 \mathrm{~m} / \mathrm{d}$ for the Kaikōura earthquake. The higher the degree of rock damage is, the higher would be the hydraulic conductivity. Laboratory tests have demonstrated that the loading-induced increase in the volumetric strain would cause the permeability to expand [45-52]. However, according to (7) and (8), a specific relationship between the rock damage and the specific storage or porosity was not found [16]. Low permeability rock may have very high porosity (>99\%) [20]. Based on the parameters for the Solomon Islands earthquake, the results of $0.293<\alpha_{\mathrm{D}}<0.363,0.226<A<0.251, K=69 \mathrm{~m} / \mathrm{d}$, and $n=0.22$ for the X10 well calculated for the Kaikoura earthquake (2016-11-13) had a lower degree of rock damage. On the day before the Solomon Islands earthquake, there was a $M \mathrm{w} 6.2$ earthquake (Hutubi earthquake, 2016-12-08) in Changji county of Xinjiang Province, China. The epicenter was located $113 \mathrm{~km}$ west of the X10 well, and there were numerous aftershocks. This earthquake destroyed the structure of the strata and altered the hydrogeological conditions of the X10 well, especially the stress-strain conditions, which led directly to higher rock damage and higher hydraulic permeability. It is concluded that using the relationship between the seismic waves and the water waves to invert the degree of rock damage is a feasible proposition. For the same aquifer conditions, there is a connection between the degree of rock damage and the aquifer properties. The higher the degree of rock damage is, the higher will be the hydraulic conductivity, and thus a method to estimate the degree of rock damage and the properties of the aquifer from water level fluctuations in wells induced by seismic waves has been realized.

\section{Conclusions}

A method for estimating the degree of rock damage and the hydraulic properties of the aquifer in the X10 well from the fluctuations in the water level induced by seismic waves from the Mw 7.8 Solomon Islands earthquake (2016-12-09) and the Mw 7.8 Kaikōura earthquake (2016-11-13) has been developed. Based on the curve fitting data, the best correlation coefficient between the calculated and measured water pressures showed that the changes in the pore pressure were influenced by both the volumetric strain and the deviatoric strain. By comparing the results with the tidal response, it was shown that the results represent the actual aquifer characteristics. In this work, the damage parameter $0.662<\alpha_{\mathrm{D}}<0.754$ for the Solomon Islands earthquake was related to the shear strain coupling coefficient $N=8.437 \mathrm{GPa}$ and the Skempton coefficient $-0.100<A<0.026$; in the case of the Kaikōura earthquake, the respective values were $0.293<\alpha_{\mathrm{D}}<0.363, N=2.529 \mathrm{GPa}$, and $0.226<A<0.251$. It was shown to be feasible to invert the value for the degree of rock damage by using the relationship between the seismic waves and the water waves. For the same aquifer conditions, there is a connection between the degree of rock damage and 
the hydraulic properties of the aquifer, namely, the higher the degree of rock damage is, the higher will be the hydraulic conductivity of the aquifer. Thus, characterization studies on the hydraulic properties of the aquifer may be used to study the degree of rock damage.

\section{Data Availability}

Requests for access to these data should be made to the corresponding author.

\section{Disclosure}

The original water level and seismic wave data supporting the work presented in this paper are from the monitoring centre of the Earthquake Agency of the Xinjiang Uygur Autonomous Region. These data are essential for this research.

\section{Conflicts of Interest}

The authors declare that they have no conflicts of interest.

\section{Authors' Contributions}

Di Zhao was responsible for methodology, formal analysis, writing, review and editing, and visualization. Yifan Zeng was responsible for writing and formal analysis. Xiaolong Sun was responsible for formal analysis and visualization. Aoshuang Mei was responsible for review and editing.

\section{Acknowledgments}

This research was supported by the National Natural Science Foundation of China (42072284, 42027801, and 41877186 to Yifan Zeng; 41972253 to Xiaolong Sun) and the Major Science and Technology Projects of Inner Mongolia Autonomous Region (2020ZD0020-4).

\section{References}

[1] J. C. Jaeger and N. G. W Cook, Fundamentals of Rock Mechanics, Science Paperbacks, LondonUK, Third edition, 1979.

[2] M. D. Zoback, Reservoir Geomechanics, Cambridge University Press, Cambridge, UK, 2007.

[3] M. Manga and C.-Y. Wang, "Earthquake hydrology," Treatise on Geophysics, vol. 4, pp. 293-320, 2007.

[4] E. Shalev, I. Kurzon, M.-L. Doan, and V. Lyakhovsky, "Sustained water-level changes caused by damage and compaction induced by teleseismic earthquakes," Journal of Geophysical Research: Solid Earth, vol. 121, no. 7, pp. 49434954, 2016.

[5] L. M. Kachanov and D. Krajcinovic, "Introduction to continuum damage mechanics," Journal of Applied Mechanics, vol. 54, no. 2, p. 481, 1986.

[6] V. Lyakhovsky, Y. Ben-Zion, and A. Agnon, "Distributed damage, faulting, and friction," Journal of Geophysical Research: Solid Earth, vol. 102, no. B12, pp. 27635-27649, 1997.

[7] P. A. Hsieh, J. D. Bredehoeft, and J. M. Farr, "Determination of aquifer transmissivity from earth tide analysis," Water Resources Research, vol. 23, no. 10, pp. 1824-1832, 1987.
[8] S. Rojstaczer, "Determination of fluid flow properties from the response of water levels in wells to atmospheric loading," Water Resources Research, vol. 24, no. 11, pp. 1927-1938, 1988.

[9] L. Xue, H.-B. Li, E. E. Brodsky et al., "Continuous permeability measurements record healing inside the Wenchuan earthquake fault zone," Science, vol. 340, no. 6140, pp. 1555-1559, 2013.

[10] Z. Shi and G. Wang, "Aquifers switched from confined to semiconfined by earthquakes," Geophysical Research Letters, vol. 43, no. 21, Article ID 11166, 2016.

[11] Z. Shi and G. Wang, "Evaluation of the permeability properties of the Xiaojiang fault zone using hot springs and water wells," Geophysical Journal International, vol. 209, no. 3, pp. 1526-1533, 2017.

[12] C.-Y. Wang and A. J. Barbour, "Influence of pore pressure change on coseismic volumetric strain," Earth and Planetary Science Letters, vol. 475, pp. 152-159, 2017.

[13] Y. Kano and T. Yanagidani, "Broadband hydroseismograms observed by closed borehole wells in the Kamioka mine, central Japan: response of pore pressure to seismic waves from 0.05 to $2 \mathrm{~Hz}$," Journal of Geophysical Research, vol. 111, no. B3, Article ID B03410, 2006.

[14] Y. Zhang, L.-Y. Fu, F. Huang, and X. Chen, "Coseismic waterlevel changes in a well induced by teleseismic waves from three large earthquakes," Tectonophysics, vol. 651-652, pp. 232-241, 2015.

[15] C.-y. Wang, Y. Chia, P.-l. Wang, and D. Dreger, "Role of S waves and Love waves in coseismic permeability enhancement," Geophysical Research Letters, vol. 36, no. 9, Article ID L09404, 2009.

[16] X. Sun, Y. Xiang, and Z. Shi, "Estimating the hydraulic parameters of a confined aquifer based on the response of groundwater levels to seismic Rayleigh waves," Geophysical Journal International, vol. 213, no. 2, pp. 919-930, 2018.

[17] H. H. Cooper, J. D. Bredehoeft, I. S. Papadopulos, and R. R. Bennett, "The response of well-aquifer systems to seismic waves," Journal of Geophysical Research, vol. 70, no. 16, pp. 3915-3926, 1965.

[18] L.-B. Liu, E. Roeloffs, and X.-Y. Zheng, "Seismically induced water level fluctuations in the Wali well, Beijing, China," Journal of Geophysical Research, vol. 94, no. B7, pp. 94539462, 1989.

[19] Y. Kitagawa, S. Itaba, N. Matsumoto, and N. Koizumi, "Frequency characteristics of the response of water pressure in a closed well to volumetric strain in the high-frequency domain," Journal of Geophysical Research, vol. 116, no. B8, Article ID B08301, 2011.

[20] E. E. Brodsky, E. Roeloffs, D. Woodcock, I. Gall, and M. Manga, "A mechanism for sustained groundwater pressure changes induced by distant earthquakes," Journal of Geophysical Research, vol. 108, no. B8, pp. 2390-2399, 2003.

[21] D. A. Lockner and S. Stanchits, "Undrained poroelastic response of sandstones to deviatoric stress change," Journal of Geophysical Research, vol. 107, no. B12, pp. 2353-2366, 2002.

[22] Y. Hamiel, V. Lyakhovsky, and A. Agnon, "Rock dilation, nonlinear deformation, and pore pressure change under shear," Earth and Planetary Science Letters, vol. 237, no. 3-4, pp. 577-589, 2005.

[23] E. Shalev, I. Kurzon, M.-L. Doan, M. Linh, and V. Lyakhovsky, "Water-level oscillations caused by volumetric and deviatoric dynamic strains," Geophysical Journal International, vol. 204, no. 2, pp. 841-851, 2016.

[24] A. Sterling and E. Smets, "Study of earth tides, earthquakes and terrestrial spectroscopy by analysis of the level fluctuations in a borehole at heibaart (Belgium)," Geophysical Journal 
of the Royal Astronomical Society, vol. 23, no. 2, pp. 225-242, 1970.

[25] T. C. Rasmussen and L. A. Crawford, "Identifying and removing barometric pressure effects in confined and unconfined aquifers," Ground Water, vol. 35, no. 3, pp. 502-511, 1997.

[26] Z. Shi, G. Wang, M. Manga, and C.-Y. Wang, "Mechanism of co-seismic water level change following four great earthquakes - insights from co-seismic responses throughout the Chinese mainland," Earth and Planetary Science Letters, vol. 430, pp. 66-74, 2015.

[27] E. A. Roeloffs, "Fault stability changes induced beneath a reservoir with cyclic variations in water level," Journal of Geophysical Research, vol. 93, no. B3, pp. 2107-2124, 1988.

[28] P. Segall, "Earthquakes triggered by fluid extraction," Geology, vol. 17, no. 10, pp. 942-946, 1989.

[29] C. R. Carrigan, G. C. P. King, G. E. Barr, and N. E. Bixler, "Potential for water-table excursions induced by seismic events at Yucca Mountain, Nevada," Geology, vol. 19, no. 12, pp. 1157-1160, 1991.

[30] D. J. Hart and H. F. Wang, "Laboratory measurements of a complete set of poroelastic moduli for Berea sandstone and Indiana limestone," Journal of Geophysical Research: Solid Earth, vol. 100, no. B9, pp. 17741-17751, 1995.

[31] D. J. Henkel and N. H. Wade, "Plane strain tests on a saturated remoded clay," Journal of the Soil Mechanics and Foundations Division, vol. 92, no. 6, pp. 67-80, 1966.

[32] R. D. Holtz, W. D. Kovacs, T. C. Sheahan, and P. Hall, Introduction To Geotechnical Engineering, An: United States Edition, Pearson Schweiz Ag, Zug, Switzerland, 1981.

[33] A. W. Skempton, "The pore-pressure coefficients A and B," Géotechnique, vol. 4, no. 4, pp. 143-147, 1954.

[34] M. A. Biot, "Theory of elasticity and consolidation for a porous anisotropic solid," Journal of Applied Physics, vol. 26, no. 2, pp. 182-185, 1955.

[35] H. F. Wang, Theory of Linear Poroelasticity, Princeton University Press, Princeton, NJ, USA, 2000.

[36] M. L. Doan and F. H. Cornet, "Small pressure drop triggered near a fault by small teleseismic waves," Earth and Planetary Science Letters, vol. 258, no. 1-2, pp. 207-218, 2007.

[37] X. Sun, G. Wang, and X. Yang, "Coseismic response of water level in Changping well, China, to the $M w 9.0$ Tohoku earthquake," Journal of Hydrology, vol. 531, pp. 1028-1039, 2015.

[38] J. D. Bredehoeft, "Response of well-aquifer systems to earth tides," Journal of Geophysical Research, vol. 72, no. 12, pp. 3075-3087, 1967.

[39] W. F. Brace, B. W. Paulding, and C. Scholz, "Dilatancy in the fracture of crystalline rocks," Journal of Geophysical Research, vol. 71, no. 16, pp. 3939-3953, 1966.

[40] T. Lay and T. C. Wallace, Modern Global Seismology, p. 521, Academic Press, Cambridge, MA, USA, 1995.

[41] P. M. Shearer, Introduction to Seismology, Cambridge University Press, Cambridge, UK, 2009.

[42] Y. Zhang and F. Huang, "Mechanism of different coseismic water-level changes in wells with similar epicentral distances of intermediate field," Bulletin of the Seismological Society of America, vol. 101, no. 4, pp. 1531-1541, 2011.

[43] X. Liao, C. Wang, and C. Liu, "Disruption of groundwater systems by earthquakes," Geophysical Research Letters, vol. 42, no. 22, pp. 9758-9763, 2016.

[44] H. F. Wang, "Effects of deviatoric stress on undrained pore pressure response to fault slip," Journal of Geophysical Research: Solid Earth, vol. 102, no. B8, pp. 17943-17950, 1997.
[45] J. Zuo, H. Xie, H. Zhou, and S. Peng, "Thermal-mechanical coupled effect on fracture mechanism and plastic characteristics of sandstone," Science in China - Series E: Technological Sciences, vol. 50, no. 6, pp. 833-843, 2007.

[46] H. Xie, J. Xie, M. Gao et al., "Theoretical and experimental validation of mining-enhanced permeability for simultaneous exploitation of coal and gas," Environmental Earth Sciences, vol. 73, no. 10, pp. 5951-5962, 2015.

[47] H. Yi, H. Zhou, R. Wang, D. Liu, and J. Ding, "On the relationship between creep strain and permeability of granite: experiment and model investigation," Energies, vol. 11, no. 10, pp. 2859-2873, 2018.

[48] H. W. Zhou, D. Liu, G. Lei, D. J. Xue, and Y. Zhao, "The creepdamage model of salt rock based on fractional derivative," Energies, vol. 11, pp. 2347-2355, 2018.

[49] X. Liu, D. Fan, Y. Tan et al., "Failure evolution and instability mechanism of surrounding rock for close-distance parallel chambers with super-large section in deep coal mines," International Journal of Geomechanics, vol. 21, no. 5, Article ID 04021049, 2021.

[50] X. Liu, S. Song, Y. Tan et al., "Similar simulation study on the deformation and failure of surrounding rock of a large section chamber group under dynamic loading," International Journal of Mining Science and Technology, vol. 31, no. 3, pp. 495-505, 2021.

[51] B. Chen, "Stress-induced trend: the clustering feature of coal mine disasters and earthquakes in China," International Journal of Coal Science \& Technology, vol. 7, no. 4, pp. 676692, 2020.

[52] X. Wang, Y. Tang, S. Wang, and H. H. Schobert, "Clean coal geology in China: research advance and its future," International Journal of Coal Science \& Technology, vol. 7, no. 2, pp. 299-310, 2020. 